\title{
Molecular biology, cell biology and NCB
}

The accelerating rate of technological advance is revolutionising molecular cell biology. Hardware advances in automation, DNA sequencing, mass spectroscopy and various forms of microarray analysis allow large-scale genomic and proteomic analyses. Bioinformatic advances allow increasingly sophisticated interrogation and management of exponentially growing datasets. Techniques such as PCR, GFP/FRET and RNAi have led to quantum leaps in experimental sophistication. These developments have resulted in a recasting of the science landscape, with an increased reliance on technology-driven core facilities at research institutes and international research consortia, as well as the availability of large datasets generated by non-hypothesis-driven research.

No single area has been affected more by these developments than molecular biology. Originally, the term was associated with the study of DNA around the middle of the last century, anchored by the dramatic symbolism of the double helix. It then evolved to include nuclear events in the broader sense, such as transcription and chromatin, framed by cell and developmental biology, genetics and biochemistry. Since then, the term has taken on an entirely new meaning. It now describes a set of essential techniques used by the majority of bioscience laboratories: for example, these days a pathology lab relies as much on molecular approaches as on modern imaging techniques, and a developmental lab will work from the traditional description of epigenetic relationships to the documentation of such pathways at the molecular level. As such, the traditional 'molecular biologist' is on the verge of extinction, not because she has succumbed to superior approaches, but rather because molecular biology has grown to permeate all walks of biology.

The molecular biology revolution is also moulding the journal landscape. Aside from the new crop of journals covering the area, established journals such as Nature Cell Biology and Nature Structural Biology have increasingly adapted to encompass the term over the years. At this time, it is worth taking stock briefly.

NSB has become increasingly focused on function, culminating this month in the announcement of a new title to better reflect this wider scope (http://www.nature.com/natstructmolbiol).

NCB has followed a complementary path by expanding its scope into the molecular domain. Importantly, this is not occurring at the expense of studies in cell biology: molecular biology essentially provides the tools required for a mechanistic understanding of cell biological phenomena. In terms of scope, we have added pages to cover fields that are very much a part of cell biology, but that traditionally are sometimes more aligned to older interpretations of the term 'molecular biology'. These include fields such as the cell cycle and DNA repair, chromatin, transcription and translation, protein degradation and folding, and signal transduction. Traditional focal points in cell biology, such as the cytoskeleton and membrane traffic, remain very much at the heart of this journal. Although the gene and the protein are the basic functional units of molecular cell biology, the cell is the functional unit of life. NCB is interested in how cells function, and this includes developing an understanding of their inner workings, but also importantly how a cell interacts with its environment and vice versa. In essence, the scope of this journal encompasses all studies that relate to cell-intrinsic biology. Note that we regard developmental and stem cell biology as within our editorial purview.

At the level of the individual paper, we strive to publish the most complete, mechanistically involved and physiologically relevant datasets possible. The advent of RNAi, for example, has added yet another 'loss-of-function' technique that makes this essential line of experimentation realistically feasible in almost any setting. Similarly, the availability of genomic sequences for most of the favoured model systems facilitate cloning and cross-species comparisons, and fluorescent protein tags allow assessment of proteins and protein interactions in their native environment.

So how do NCB and NSB fit into the molecular landscape together? NCB favours studies with an emphasis on physiological relevance at the cellular and organismal level. Nevertheless, we have a penchant for mechanism, which may require in vitro and indeed biophysical approaches. NSB approaches molecular mechanistic insight from a complementary angle by emphasizing advances at the detailed molecular level, often on the basis of structure-function relationships. Think in vivo and micron for $\mathrm{NCB}$, and in vitro and angstrom for NSMB. Together, we hope to cover the whole spectrum of molecular biology.

\section{IMAGING IN CELL BIOLOGY}

Enclosed with this issue is a supplement on imaging in cell biology that has been produced in conjunction with Nature Reviews Molecular Cell Biology. This series of specially commissioned articles was selected in consultation with experts in the community. The topics chosen aim to highlight the diverse imaging techniques that have become so essential for addressing key cell biological questions. The supplement will be accompanied by an online web focus that will be freely accessible for six months. In addition to the articles contained within the supplement, the web focus draws together a collection of research papers and reviews from across Nature Publishing Group that provide particularly compelling examples of imaging in cell biology. We hope that this collection of papers provides a useful resource for researchers who want to keep up with this rapidly evolving field. 